\title{
CRYSTALS AND PHOTONS
}

$I^{N}$ his presidential address to the Indian Academy of Science, Sir C. V. Raman surveyed recent progress in the investigation of those phenomena which are observed when radiation is scattered in a crystal (Current Science, January 1941. Supplement). The subject is a natural extension of the well-known Raman effect usually observed in liquids, which has proved such a fruitful souree of knowledge concerning molecular vibrations.

The analogous effects observed in crystals are, according to Raman and his collaborators, the result of interaction between the incident radiation and the material particles of the crystal. As a result of the absorption of energy from the incident beam, the regular geometrical array of atoms or molecules in the crystal is set into vibration. The lattice waves in the crystal can then be regarded as stratifications of density which may reflect the energy preferentially in certain directions. Owing to the interchange of energy between radiation and matter, the reflected ray suffers a change of wave-length. Thus when a diamond is illuminated by monochromatic radiation, the scattered ray contains two components with wave numbers differing by $\pm 1332 \mathrm{~cm} .^{-1}$ from the wave number of the incident ray. This change of frequency appears on the quantum theory as the result of an exchange of energy between a photon and the crystal, a diminution occurring if the photon is the donor and the crystal the acceptor, an increase if the reverse is the case.

In the experiment just described, the radiation is scattered in all directions from the crystal ; but Sir C. V. Raman also envisages circumstances in which the incident radiation is regularly reflected in certain directions. He states that infra-red radiation, within a certain range characteristic of the material, is strongly reflected backwards by the crystal because the optical vibrations of the lattice are powerfully excited by the incident waves. Recently, the observation of similar phenomena has been extended to the range of X-ray frequencies. According to Raman, the optical vibrations of the lattice, excited by the incident $X$-rays, create dynamic stratifications of the electron density, and these give regular reflexion of $X$-rays with a change of frequency in much the same way that the static planes of atoms give the classical Bragg reflexions without change of frequency.

These ideas are obviously of great interest, especially with regard to the reflexion of X-rays. This part of the subject has received considerable attention in both Europe and the United States. Reference to the correspondence columns of NATURE in the last year or two shows that the X-ray phenomena which Raman describes have been ascribed by other in vestigators to reflexion from the thermal waves in the lattice. The difference in point of view seems to be that Raman considers the lattice waves to be a result of excitation by the incident energy, and he also postulates a frequency change in the scattered energy. His case would be greatly strengthened by direct observation of this frequency change, which is, unfortunately, too small to be detected at present. The other approach to the problem regards the lattice waves as being already present in the crystal as a result of the thermal vibrations of the atoms, and the observed effects do, in fact, appear to be explicable on these lines. Whether a quantum mechanism is necessary for an accurate description of the phenomenon would seem to be a matter for still further investigation (see also p. 467 of this issue).

\section{DAMS AND THE PROBLEM OF MIGRATORY FISHES}

$\mathrm{T}$ HE special symposium issue, with the above title, of the Stanford Ichthyological Bulletin, published through the co-operation of the Fish Commission of the State of Oregon and the Natural History Museum of Stanford University, comprises a number of papers read by leading fishery authorities in the United States on the problem presented by the construction of dams for generating electric power and for irrigation of agricultural land in certain States on the Pacific seaboard of North America.

The dams in question-already constructed or to be constructed-are in the basins of the Columbia and Sacramento Rivers. The fish mainly affected are the anadromous Pacific salmon (Oncorrhyncus) - chinook, blue back and cohoe-and the steel-head trout; fish which are of great importance in connexion with the world-famed commercial fisheries of the North American Pacific coast. Consideration has had to be given to the question of the effect these dams will have not only on the ascent of the breeding fish to their customary spawning grounds (and on the spawning grounds themselves) but also on the descent of the fry (and spent aduits).

Fundamentally, a proposition of this nature re- solves itself into the formulation of two questions, namely, (a) are such industrial developments on a river compatible with the continued maintenance of the fisheries in that river, and $(b)$ if the answer is in the negative, are the fisheries to be regarded as of secondary importance and therefore inevitably to be sacrificed-the issue being the relative merits of each $q u \hat{a}$ progress? A subsidiary question will be the cost of the necessary measures involved under $(a)$ to safeguard the fishery interests. If the cost is high it may be urged that the proposition really comes under heading $(b)$.

At the outset neither of these questions seems to have even suggested itself to the industrial interests bent on exploiting these rivers as power generators. Nor does it appear that the central authorities vested with the power of supervisory administration (and whose consent had to be obtained in the first instance) were any more alive to the situation and to its im. plications. It devolved upon the fishery interests, through the American Bureau of Fisheries, to direct attention to the probable effect on the fisheries and to the very considerable monetary value to the community at large of those fisheries. Inciden- 